\title{
Counseling to Prevent Obesity Among Preschool Children: Acceptability of a Pilot Urban Primary Care Intervention
}

\author{
M. Diane McKee, MD, MS \\ Stacia Maber, MPH \\ Darwin Deen, MD, MS \\ Artbur E. Blank, PbD
}

Department of Family and Social Medicine, Albert Einstein College of Medicine, Bronx, New York
Conflicts of interest: none reported

\section{CORRESPONDING AUTHOR}

M. Diane McKee, MD, MS

Department of Family and Social Medicine 1300 Morris Park Ave

Albert Einstein College of Medicine

Bronx, NY, 10461

mckee@aecom.yu.edu

\begin{abstract}
PURPOSE To help design effective primary care-based interventions, we explored urban parents' reactions to a pilot and feasibility study designed to address risk behaviors for obesity among preschool children.
\end{abstract}

METHODS We conducted 3 focus groups (2 in English, 1 in Spanish) to evaluate the pilot intervention. Focus group participants explored the acceptability of the pilot intervention components (completion of a new screening tool for risk assessment, discussion of risk behaviors and behavior change goal setting by physicians, and follow-up contacts with a lifestyle counselor) and the fidelity of the pilot intervention delivery.

RESULTS Parents expressed a desire to change behaviors to achieve healthier families. They believed that doctors should increase their focus on healthy habits during visits. Parents were more accepting of nutrition discussions than increasing activity (citing a lack of safe outdoor space) or decreasing sedentary behaviors (citing many benefits of television viewing). Contacts with the lifestyle counselor were described as empowering, with parents noting her focus on strategies to achieve change for the whole family while recognizing that many food behaviors relate to cultural heritage. Parents expressed frustration with physicians for offering advice about changing behavior but not how to achieve it, for dismissing concerns about picky eating or undereating, and in some cases for labels of overweight that they believed were inappropriately applied.

CONCLUSIONS Parents welcomed efforts to address family lifestyle change in pediatric visits. The model of physician goal setting with referral for behavior change counseling is highly acceptable to families. Future interventions should acknowledge parental concerns about undereating and perceived benefits of television viewing.

Ann Fam Med 2010;8:249-255. doi:10.1370/afm.1057

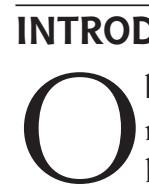

\section{ODUCTION}

besity in children is an increasing public health concern. Currently, national estimates indicate that $36 \%$ of the pediatric population is overweight or obese. ${ }^{1,2}$ Inner-city minority populations are disproportionately affected. ${ }^{3,4}$ For example, $43 \%$ of New York City's elementary public school children are overweight or obese. ${ }^{5}$ Although nutrition is one of the most common topics addressed by pediatricians during health maintenance visits of children aged 2 to 5 years ${ }^{6}$ and is addressed in at least $40 \%$ of these visits, ${ }^{7}$ the role of the primary care clinician in the prevention of childhood obesity has not been demonstrated.

Prevention is more likely to be effective when the family is the focus of the intervention ${ }^{8}$ and when nutrition education, increased physical activity, and decreased sedentary behaviors are addressed. ${ }^{9}$ Starting from this premise, we piloted an intervention aimed at promoting behavior 
change for families, targeting health maintenance visits of children aged 2 to 4 years. The Family Lifestyle Assessment of Initial Risk (FLAIR) project was 1 of 10 initiatives funded by the Robert Wood Johnson Foundation's Prescription for Health program aimed to address multiple behavioral risks in the primary care setting. Our approach was based on a socioecological model $^{10}$ that targeted families of young children by addressing lifestyle behaviors known to place children at risk for overweight. ${ }^{11}$

We report the results of a series of focus groups with parents conducted as part of the evaluation strategy of the FLAIR pilot intervention. The evaluation focused on 4 aims: (1) to explore caregivers' perceptions of the importance of family behavior change (2) to assess the acceptability of the pilot intervention components $;$ (3) to assess the usefulness of the pilot intervention in facilitating behavior change; ${ }_{i}$ and (4) to assess the fidelity of the pilot intervention.

\section{Overview of the FLAIR Pilot and Feasibility Study}

The pilot intervention was based in 3 hospital-affiliated primary care health centers staffed by primary care physicians (family physicians, pediatricians, and internists), located in the central or south Bronx, New York Clinic staff were oriented to the pilot intervention and instructed to give a FLAIR screening form to caregivers of children aged 22 to 59 months when preparing them for physical examinations. They introduced the screen as a new form that would help identify unhealthy behaviors. Physicians participated in workshop-style, case-based training to provide evidencebased guidelines for targeting key behavioral risks and to acquire skills in brief behavior change counseling.

The pilot intervention (Figure 1) was organized around the elements of the National Cancer Institute's $5 \mathrm{~A}^{\prime}$ s mode ${ }^{12}$ : ask, assess, advise, assist, arrange. Parents or guardians (hereafter referred to as parents) were first asked to complete the FLAIR screening questionnaire, which is a self-administered screening form, and later asked by physicians whether they were interested in learning ways they could reduce their family's risk for future health problems. The physicians assessed the family lifestyle risk according to the responses on the form and assessed the parents' readiness to change by using the simple concepts of perceived importance and confidence to change behaviors ${ }^{13}$; they then negotiated agreement on a behavior change goal with parents.

The physicians were trained to advise parents by providing specific brief counseling about the behavior selected for change. They assisted parents by offering visits with lifestyle counselors to provide more in-

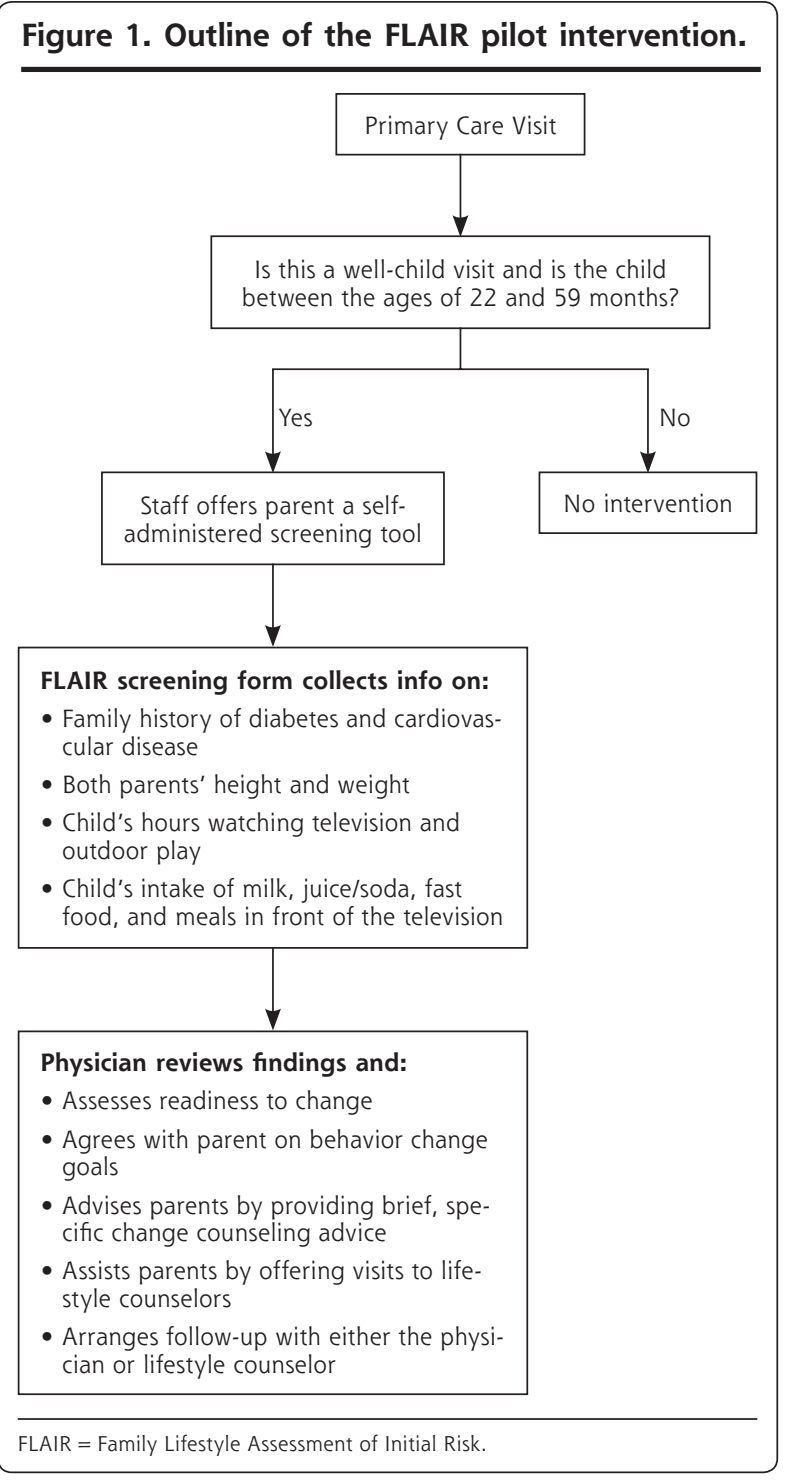

depth counseling based on motivational interviewing. ${ }^{14}$ Follow-up was arranged with either the physician or the lifestyle counselors to reinforce behavior change and identify additional behavior change goals.

Lifestyle counselors had completed health educator training and were experienced bilingual motivational interviewers. Counselors were available in each practice for 1 half-day each week during the pilot intervention. In sessions that typically lasted 1 hour, lifestyle counselors worked with parents to identify behavioral strategies to achieve the goals that were set during the well-child visit.

\section{METHODS}

The focus group evaluation was approved by the institutional review board of the authors' institution. 


\section{Recruitment}

After the pilot intervention and feasibility study were complete, to evaluate the study, we recruited parents to participate in focus groups from among families who had completed the FLAIR screening questionnaire during a preventive care visit for a child aged 22 to 59 months. Letters from their physicians were mailed to 231 parents (identified by electronic medical records and chart review) who met these criteria. Telephone calls were then made from a random-ordered list to 167 families: 93 (56\%) could not be reached at any available telephone number, $46(27 \%)$ refused to participate, 28 indicated they would attend 1 of the groups, and 18 actually attended. A $\$ 50$ money order, dinner, and childcare package was offered as an incentive to participate in a focus group; families did not receive an incentive for participating in the pilot intervention itself.

\section{Focus Group Facilitator Guide}

The focus group guide addressed the following areas: (1) the perceived importance of family behavior change (eg, Do you feel this should be a part of the medical visit?) $)_{i}(2)$ the acceptability of the pilot intervention components of completing the form, discussing the questionnaire, goal setting, and visits to the lifestyle counselor (eg, How did you feel about filling out the form? How did it feel to talk about these issues with the doctor?); (3) the usefulness of the pilot intervention in facilitating behavior change (eg, What types of health habits were changed?); and (4) the fidelity of the pilot intervention (eg, What did the nursing staff say when they introduced the form to you? What sort of discussion took place between you and your doctor? Who set the goal, you or your doctor?). Prompts addressed parents' emotional and cognitive responses to the process.

\section{Data Collection}

We conducted 3 focus groups, 2 in English ( $\mathrm{n}=5$ and $\mathrm{n}=8)$ and 1 in Spanish $(\mathrm{n}=5)$ between April and October 2007. Parents provided written informed consent before participating. The same experienced bilingual facilitator conducted all 3 focus groups. The sessions were audio-recorded and transcribed verbatim. A research coordinator observed and took detailed notes.

\section{Data Analysis}

The analysis team included 2 family physician investigators-a master's level project coordinator trained in health education, and a social psychologist. The team used an editing approach whereby the analysts independently identified key themes related to the content areas of interest. ${ }^{15}$ A preliminary coding scheme was created based on these initial themes, which was applied and refined through an iterative process in group discussions. The resulting final codes were applied to all 3 transcripts. At least 2 analysts coded each transcript. Minimal discrepancies (primarily omission of codes) were explored in meetings until consensus was achieved. ${ }^{15}$ We then searched for convergence and divergence of themes across groups. ${ }^{16,17}$

\section{RESULTS}

\section{Characteristics of Focus Group Attendees}

Focus group participants' characteristics were obtained from the registration data. With the exception of 1 father, all participants were mothers, and their mean age was 28 years. Table 1 summarizes the characteristics of the practice patients and focus group participants. Participants reflected the population of health center users; most were Hispanic and insured by Medicaid. All participants had visits during the pilot intervention period and completed the FLAIR screening questionnaire as part of their child's visit; 12 (44\%) families had documented goal setting, and 8 (44\%) also attended at least 1 session with the lifestyle counselor.

\section{Achieving Healthy Families: Frustrations With Primary Care}

Importance of Addressing Healthy Diet

The parents described a commitment to their families' health and to improving intake of healthy foods. This commitment was evident in the many strategies they had used to improve diet within the family and the high level of interest in more help from physicians. They described their experience with primary care as providing very little attention to nutrition before the FLAIR pilot intervention:

[What was different from your last visit?] I like the fact that now [my doctor] and the nurse were asking me questions

\begin{tabular}{lcc|}
\hline $\begin{array}{l}\text { Table 1. Characteristics of Practice Patient } \\
\text { Population and Focus Group Participants }\end{array}$ \\
\hline $\begin{array}{ccc}\text { Practice Users } & \text { Focus Group } \\
\text { No. (\%) }\end{array}$ \\
\hline Characteristic & & \\
\hline Insurance & & \\
Self-pay & 4 & $(11)$ \\
Medicaid & 63 & $12(67)$ \\
Medicare & 7 & $0(0)$ \\
Commercial & 20 & $4(22)$ \\
Ethnicity & & \\
White & 4 & $0(0)$ \\
Black & 22 & $4(22)$ \\
Hispanic & 68 & $14(78)$ \\
Other & 6 & $0(0)$ \\
\hline Insurance reflects status for parent. & \\
\hline
\end{tabular}


about his diet since the last visit he's had... they didn't really ask me before.

I've never [before] been asked about positive eating habits, and I guess if their weight is fine and height is fine...then they don't really go any further.

\section{Feeling Unheard by Physicians}

Some parents indicated that special effort had been needed to get their nutrition concerns addressed during visits. Children's visits were described as rushed and focused on the limited objectives of the physician:

We wanna know about, like, regular [feeding]...because if you're not on WIC [Special Supplemental Nutrition Program for Women, Infants, and Children], sometimes they don't tell you what vegetables you're supposed to give to them. When you go to the appointment, the doctor says, "Oh, let me see in your ear. Let me see your eye, your throat...fine, you're okay, bye," boom.

One area that many parents found especially frustrating was a perceived lack of attention to their concerns about underweight or picky eating:

I complained to her, I was trying to find shortcuts and tricks to get him to eat. And she was like...I shouldn't worry so much about what he's eating... because he's fine, he's growing. But I understand he's growing.... But I want him to be able to eat, you know, like other kids.

Families were also unhappy when physicians had given advice to change behaviors without help in how to make change:

[My daughter] went to the doctor 10 months ago. And the doctor told me that she's small for 4 years old. I said that it's difficult to get her to eat certain kinds of food...she'll eat a little of this, little of that, and then she leaves it. But the doctor told me, oh, it's not good, she's not eating. I should give her some PediaSure. But I was upset because... if she's not eating the food what makes him think she's going to drink the PediaSure?

\section{Discomfort With the Obesity Label}

Although parents very much appreciated having their nutrition concerns addressed, some parents expressed discomfort or even resistance to the diagnosis of obesity for their child. Some offered arguments that it is in the child's nature to be big:

My doctor wanted [him to see a nutritionist] because he was overweight, and I said, "Well, wait! First of all, let's go back to when he was born. By the time he was 3 months old, 4 months, he looked like he was 9, 10 months."

Others suggested that the diagnosis is incorrect because the charts used by physicians are wrong ("He's been off that baby chart from the day he was born.") and that physicians' expectations for weight are wrong:

My doctor told me he's double his weight for his age.... I'm thinking, he's a little overweight.... They expect a 5 -yearold to be like 20 pounds or something...? Some kids are too skinny....and then they look like crack kids.

\section{Acceptability and Fidelity of the FLAIR Pilot Intervention}

FLAIR Questionnaire: Getting Nutrition on the Agenda

Families all agreed that assessing health behaviors should be part of well-child visits. They described the screening questionnaire as easy to complete and something that should continue. One mother indicated that the questionnaire was a way of getting nutrition on the physician's agenda when it may otherwise not be:

I think [the screening form] is good. Like because most of the time the only thing I have with my doctor is... I have to ask her for what I want her to do. So like if you have this [form], and maybe you say yes to one of these things, and maybe she would give you more information and help you better based on the information that you have [on the form].

Parents emphasized the importance of the doctor's involvement in the screening process. They viewed the screening form as a tool for the physician "to get to know you" and to increase communication with the physician:

I think that [the screening form] is necessary because you can have better communication with the doctor. You find yourself more prepared [to ask questions].

\section{Fears of Being Judged}

Parents did express some concerns about the process of reporting health behaviors. Although only a few indicated less than completely truthful answers, several expressed fear of being judged or appearing neglectful:

Nobody discussed it with me...no one sat down and explained it to me.... I don't want her to feel that I'm doing anything neglectful towards [my son].

\section{Focusing on Family}

Most parents were comfortable with the emphasis on family lifestyle change. In contrast, however, this mother was not comfortable shifting the focus away from the individual child toward the entire family:

[My child's doctor said,] "Look at you, you're overweight for your size." And I almost felt like saying this is not about me. This is about him. When I go see my doctor, I'll let her 
tell me what, you know, that I am (overweight). But I just felt that that made me feel so crappy.... I was asking for help for my son, not for me.

Some parents did not make the connection between parent's behaviors and the child's weight:

They gave me a lot of papers... how to eat and how I was going to cook... It was hard. I feel like they wanted to change me..., but I know it wasn't for me, it was for my fat son.

\section{Physician-Facilitated Goal Setting}

The process of setting goals with physicians was well received. Participants reported setting such goals as decreasing fast food, decreasing sweetened beverages, increasing activity, and decreasing television. Consistent with our goal of facilitating a patient-centered process, most, but not all, indicated they had set goals together with the physician in a mutual process. In general, parents were most receptive to setting goals related to nutrition but much less ready to address television viewing. Mothers were offended by the suggestion of laziness ("It almost makes you feel like [they think] you are not doing anything."). Mothers noted the many benefits of television, including its calming effect on children ("If you shut it off, he's going to slam everything around.), education (eg, improved language skills), and keeping the child occupied: "So if I am cooking dinner, I have to have something for her to do. But she's watching Dora...where she's learning something."

\section{Empowering Parents: Lifestyle Counselor Contacts} Parents' overall impression of the lifestyle counseling was very positive, describing it as something they would recommend to other families. Descriptions suggest that the counseling closely followed the motivational interviewing approach we sought. Mothers understood the counselor's role as an empowering resource, focused on strategies, not just advice, to change behavior:

[The health educator's role was] to help you make better decisions, not only in eating but in changing your lifestyle. Because she was not-you know people think that diets are going to be quick. She goes, it's a lifestyle change.... And I'm here to help you... That's why we had you fill out this stuff-so you can see where you are, and I can show you how to get where you want to be.

Participants found it helpful that the counselor did not suggest that behavior change was all or nothing, but rather viewed it as a stepwise process:

She said it's not going to be easy. She goes, you're going to get mad sometimes. You're going to want to cheat. She goes, it's all right to cheat.... She also said, you know, if you happen to eat a lot of fast foods, McDonalds now has healthy options. You have salads, you know. For the kids you don't have to get them French fries. They have those little apple things and they can get milk.

Mothers appreciated that the counselor provided a variety of tools, often providing pamphlets, recipes, or Web sites. She helped identify community resources, such as farmer's markets, but most importantly she spent time strategizing with parents about how to overcome barriers to change.

I told her it is not easy to change in my house, because there are 2 families there. She asked, "What are you doing with him now, what tricks do you use to get him to eat vegetables...?" She goes, "You really don't need that much help. You know this stuff, you shouldn't worry."

Facilitating Behavior Change in the Urban Context Most families indicated they had made changes as a result of the physician's or health educator's advice. Of note, several examples showed that our goal of focusing at the family rather than the individual level was achieved. This participant indicated that behaviors of older children in the house changed even though the initial focus had been on the younger child:

At first when I first, like, told the kids about it, they were like, "Well, why we can't eat [McDonald's]? You and her can't eat it, but why can't we eat it?" Then I said, "Because if you come in here with McDonald's and she sees it...everybody's going to want it...." So I said, "Instead, why don't we all just think about it together..."

Examples of behavior change efforts highlighted the many barriers urban families face. Parents noted lack of safe or accessible play areas and poor availability of fresh foods. Parents emphasized that the family food environment was not always entirely under their control. Multigenerational households were a common source of difficulty:

[So you eliminated the fast food, you eliminated the juice, and everyone made these changes?] Yes, except my mother... It's that she has this tiny fridge [full of junk food!] in her room! You enter...grab a juice, a soda, whatever things that you want in this little fridge. At 3 or 4 [the children] are hungry, she gives them food. I'm like, oh, what are you doing?

\section{DISCUSSION}

Many primary care physicians address nutrition and exercise behaviors at least briefly during most pediatric preventive visits. ${ }^{6,7}$ The FLAIR pilot intervention introduced a process designed to address risk factors based on the 5 A's model of the National Cancer Institute. ${ }^{12}$ The self-administered screening form accomplished the ask step. The physicians provided advice regarding the various behaviors and risk of excessive weight gain. 
They then assessed by reviewing the forms and helping to set goals with the parents. Assistance was provided through referral to the health educator, whose role it was to arrange follow-up with the patient. The FLAIR pilot intervention was preventive, encouraging behaviors that decrease risk, rather than focusing on children who were already overweight.

Our findings from the focus group evaluation of this pilot intervention indicate that the primary care office is a legitimate entry point for intervening in the problem of childhood overweight. Families welcomed a change from brief directive advice to family-centered goal setting for obesity-related risk behaviors. The data presented here indicate that mothers of preschool children in our urban setting are very interested in improving their children's intake of healthy foods, value the involvement of physicians, and find sessions with a skilled behavior change counselor useful and acceptable. Although preliminary, our data suggest that behavior change occurred only as a result of a physician raising the issue and referring to a highly skilled counselor, who then spent time with families to identify unique strategies to overcome barriers.

Recent literature reviews indicate that familyfocused interventions starting at a young age have the best chances for successfully addressing overweight in childhood. ${ }^{8}$ The FLAIR pilot intervention was designed to be a family-focused socioecological approach. ${ }^{10}$ As expected, the initial focus in the examining room was between the physician and the child's parent. The lifestyle counselor had success in moving the focus from this dyad to the broader socioecological context (simplistically, the family and social context in which health behaviors are embedded) as she identified facilitators and barriers to lifestyle change. Strategies for change necessarily were derived and implemented in this broader context. Even though the family focus was generally accepted, some families were less able to make a connection between children's behaviors and adult health; thus, these conversations may require more direct discussion.

Some issues were encountered that should be considered in the design of future interventions. We noted the potential to offend parents by both the tone and content of health behavior change messages. Importantly, we noted the strong commitment of parents to the health of families. Sometimes, however, overwhelming barriers to change are rooted in the low-income urban context, such as complex family dynamics around control of resources and generational conflict about food. Parents expressed frustration with physicians for offering advice about what to do but not how to achieve it, for dismissing their concerns about picky eating or undereating, and in some cases for labels of overweight they believed were inappropriately applied. From this response, we conclude that behavior change discussions must acknowledge the positive rather than the negative aspects of the family dynamic, give credit for positive behaviors, and focus on strengths that families can build on.

These results indicate that physicians need help to deliver health behavior change messages effectively. Specifically, training for future practice-based interventions must recognize parents' concern about picky eating and enhance the physicians' skills in addressing socially normative eating behavior. Additionally, messages about changing television viewing must be presented carefully. Parents bring a very different perspective to television viewing than do physicians, noting the many benefits and few risks. Parents perceived advice to decrease the time spent watching television as implying poor parenting or laziness. In a future intervention we would at least drop the assessment of parental television viewing. Rather than focusing on screen time, we should change the primary message about television to helping parents become aware of the undesirable commercial messages about unhealthy food that are seen on the television.

Several limitations to this study must be noted. Recruitment was restricted to families who had visits during the pilot intervention period and completed the FLAIR screening questionnaire; recruitment was further limited because a large proportion of such families could not be reached by telephone. Thus our sample does not include families who may have either been unable or chose not to complete the screening form. Parents who participated may also differ from nonparticipants in that they could be reached at available telephone numbers, and their choice to participate in the groups may reflect greater commitment to behavior change; thus, they may not be typical of all families receiving care in urban health centers. Because our evaluation was retrospective, there was delay between visits to the health center and the focus group discussion, which may have affected recall for some participants. These issues may be diminished in future studies by recruiting for evaluation efforts at the time of the visit. In addition, resources for this project limited the number of groups that could be held. Although the facilitator was not a physician, some reluctance to criticize physicians and participants' health centers could have influenced the discussion.

This project showed the overall acceptability of an approach that incorporates health behavior change into preventive care for preschool children. The model we used of physician goal setting with referral for behavior change counseling is highly acceptable to families. An overarching lesson relates to the need in 
this urban setting to focus behavior change messages toward raising healthy children rather than preventing obesity. Parents are supportive of the effort to promote healthy behaviors - important for all children and their families-but may not share physicians' definitions of overweight. Future interventions should in particular acknowledge parental concerns about undereating and perceived benefits of television viewing.

To read or post commentaries in response to this article, see it online at http://www.annfammed.org/cgi/content/full/8/3/249.

Key words: Obesity; overweight; health promotion; dietary modification; minority

Submitted January 14, 2009; submitted, revised, July 8, 2009; accepted August 3, 2009.

Funding support: This research was supported by the Robert Wood Johnson Foundation Prescription for Health.

A preliminary version of these findings was presented at the Annual Meeting of the North American Primary Care Research Group, November 2008, Rio Grande, Puerto Rico.

Acknowledgments: The authors thank the Prescription for Health National Advisory Committee for their assistance throughout the project.

\section{References}

1. Ogden CL, Carroll MD, Curtin LR, McDowell MA, Tabak CJ, Flegal KM. Prevalence of overweight and obesity in the United States, 1999-2004. JAMA. 2006;295(13):1549-1555.

2. Nicklas TA, Baranowski T, Cullen KW, Berenson G. Eating patterns, dietary quality and obesity. J Am Coll Nutr. 2001;20(6):599-608.

3. O'Loughlin J, Maximova K, Tan Y, Gray-Donald K. Lifestyle risk factors for chronic disease across family origin among adults in multiethnic, low-income, urban neighborhoods. Ethn Dis. 2007;17(4): 657-663.
4. Mirza NM, Kadow K, Palmer M, Solano H, Rosche C, Yanovski JA. Prevalence of overweight among inner city Hispanic-American children and adolescents. Obes Res. 2004;12(8):1298-1310.

5. New York City Youth Health Coalition. Healthy Kids Healthy Futures: A Resource Guide 2004. New York, NY: New York City Youth Health Coalition; 2004.

6. Olson LM, Inkelas M, Halfon N, Schuster MA, O'Connor KG, Mistry R. Overview of the content of health supervision for young children: reports from parents and pediatricians. Pediatrics. 2004;113 (6)(Suppl):1907-1916.

7. Barkin SL, Scheindlin B, Brown C, Ip E, Finch S, Wasserman RC. Anticipatory guidance topics: are more better? Ambul Pediatr. 2005;5(6):372-376.

8. St Jeor ST, Perumean-Chaney S, Sigman-Grant M, Williams $C$, Foreyt J. Family-based interventions for the treatment of childhood obesity. J Am Diet Assoc. 2002;102(5):640-644.

9. Steinbeck K. Childhood obesity. Treatment options. Best Pract Res Clin Endocrinol Metab. 2005;19(3):455-469.

10. Robinson T. Applying the socio-ecological model to improving fruit and vegetable intake among low-income African Americans. J Community Health. 2008;33(6):395-406.

11. US Health and Human Services. Small Steps Kids. http://small step. gov. Accessed Oct 10, 2008.

12. Goldstein MG, Whitlock EP, DePue J; Planning Committee of the Addressing Multiple Behavioral Risk Factors in Primary Care Project. Multiple behavioral risk factor interventions in primary care. Summary of research evidence. Am J Prev Med. 2004;27(2)(Suppl):61-79.

13. Keller VF, White MK. Choices and changes: a new model for influencing patient health behavior. J Clin Outcomes Manag. 1997;4(6): 33-36.

14. Rollnick S, Miller WR, Butler CC. Motivational Interviewing in Health Care. New York, NY: Guilford Press; 2008.

15. Miller WL, Crabtree BF. Qualitative analysis: how to begin making sense. Fam Pract Res J. 1994;14(3):289-297.

16. Polit DF, Beck CT. Nursing Research: Generating and Assessing Evidence for Nursing Practice. 8th ed. Philadelphia, PA: Wolters Kluwer Health/Lippincott, Williams \& Wilkins; 2008.

17. Miles MB, Huberman AM. Qualitative Data Analysis: An Expanded Sourcebook. 2nd ed. Thousand Oaks, CA: Sage Publications; 1994. 